\title{
Application of "Click” Chemistry in Solid Phase Synthesis of Alkyl Halides
}

\section{Diparjun Das, Tridib Chanda and Lalthazuala Rokhum*}

\author{
Department of Chemistry, National Institute of Technology Silchar, Silchar-10, Assam, India \\ * Corresponding author: E-mail: lalthazualarokhum@gmail.com \\ Tel.: +913842 242915; fax: +91 3842-224797
}

Received: 28-02-2015

\begin{abstract}
A convenient and highly selective microwave assisted procedure for the conversion of allylic, benzylic and aliphatic alcohols to their corresponding halides using polymer-bound triphenylphosphine and iodine is presented. In case of symmetrical diols, mono-iodination product is obtained in very high yield. Additionally, highly regioselective behavior is observed in our procedure. Simplicity in operation, no column chromatography required for the purification of the products, recyclability of the reagents used, short reaction times and good to excellent yields are the advantages of our protocol. Most functional groups remain unaffected under our reaction condition.
\end{abstract}

Keywords: Iodination, polymer-bound triphenylphosphine, solid phase synthesis, benzylic alcohols, aliphatic alcohols, site-selective.

\section{Introduction}

Symmetrical diols are important synthons in organic synthesis and selective halogenation of one of the two chemically-equivalent hydroxyl groups of the diols with general formula $\mathrm{HO}-\left(\mathrm{CH}_{2}\right)_{\mathrm{n}}-\mathrm{OH}$ remains an exciting challenge for synthetic organic chemistry. Ironically, in spite of its immense importance, only a limited number of such protocols are reported. ${ }^{1}$ Frequently, though unfortunately, treatment of these diols with a stoichiometric amount of reagents aimed at forming the derivative of one hydroxyl functionality always results in the formation of a 1/2/1 mixture of unreacted diol, the mono- and bis-halogenation products, respectively. ${ }^{2}$ To overcome the problem associated with statistical distribution, a large excess of the starting diol is often used. This process substantially reduces the amount of bis-halogenated product, but one is then faced with the problem of separating the desired product from the bulk of unreacted diols. Another method which has been devised to surpass this inherent problem is the 'high dilution' technique where a huge amount of solvent is required. But this method is neither economically nor environmentally viable as it involves a lot of waste of solvents which are discarded in the environment. Therefore, a mild and green method which requires only stoichiometric reagents and minimum solvent in the process of iodination remains highly desirable.
Alkyl halides are indispensable intermediates and important building blocks that can be easily converted into a variety of other functional group through functional group manipulation. ${ }^{3}$ Both alkyl bromides and iodides serve as intermediates in a wide variety of reactions which make the conversion of alcohols into the corresponding halides a very important transformation in organic synthesis. ${ }^{4,5}$ The most common precursors to alkyl halides are alcohols, and their conversion into alkyl halides is a frequently encountered functional group-transformation reaction. Among the halides, iodides are the most reactive, and often show unique reactivity pattern. ${ }^{6}$ Numerous methods for the transformation of alcohols into its corresponding iodides were reported in literature, including use of $\mathrm{BF}_{3}-\mathrm{Et}_{2} \mathrm{O} / \mathrm{NaI},{ }^{7} \mathrm{P}_{2} \mathrm{I}_{4}{ }^{8} \mathrm{P}_{4} / \mathrm{I}_{2},{ }^{9} \mathrm{Cl}_{2} \mathrm{SO}-\mathrm{DMF} / \mathrm{KI},{ }^{10} \mathrm{MgI}_{2},{ }^{11}$ $\mathrm{HI},{ }^{12} \mathrm{ClSiMe}{ }_{3} / \mathrm{NaI}^{13}{ }^{13} \mathrm{R}_{3} \mathrm{PI}_{2}-\mathrm{Et}_{2} \mathrm{O}$, or $\mathrm{C}_{6} \mathrm{H}_{6} / \mathrm{HMPA},{ }^{14}$ $\mathrm{PPh}_{3} / \mathrm{DDQ} / \mathrm{R}_{4} \mathrm{~N}^{+} \mathrm{X}^{-15} \mathrm{KI} / \mathrm{BF}_{3} \cdot \mathrm{Et}_{2} \mathrm{O},{ }^{16} \mathrm{CsI}^{15} \mathrm{BF}_{3} \cdot \mathrm{Et}_{2} \mathrm{O},{ }^{17}$ $\mathrm{KI} / \mathrm{SSA},{ }^{18} \mathrm{NaI} / \mathrm{Amberlyst},{ }^{19}$ polymer-bound triphenylphosphine reagent systems. ${ }^{20}$ The existing reported methods suffer from drawbacks such as high temperature, ${ }^{21}$ long reaction times, ${ }^{22}$ low yields, drastic conditions, noncommercially available materials and tedious work-up procedures. $^{23}$ Thus introducing new methods taking place under mild conditions with higher efficiency and selectivity, lesser toxicity of reagents, shorter reaction times, easy handling and also using inexpensive and commer- 
cially available materials are still a challenge for the synthetic organic chemists.

The increased demand for microwave assisted organic synthesis reactions is due to their short reaction times and expanded reactivity range. Microwave heating is very convenient to use in organic reactions. The heating is very specific and instantaneous. The beauty of the reaction is that it does not require any contact between the reaction vessel and the energy source. It can be concluded that all the previous conventionally heated reaction can be easily performed by microwave heating. ${ }^{24}$ Many microwave assisted solid phase reactions have been reported in recent years which may include cellular uptake, and cytotoxicity studies of cymantrene-peptide bioconjugates ${ }^{25}$ solid-phase synthesis of 5-arylhistidines via a microwave-assisted Suzuki-Miyaura cross-coupling, ${ }^{26}$ synthesis of 1,2,4-oxadiazoles using polymer-bound triphenylphosphine reagent. $^{27}$

Recently solid-phase organic syntheses (SPOS) is evolving as a means to facilitate the manipulation of compound libraries via combinatorial chemistry. ${ }^{28}$ The important features of solid-phase synthesis such as purification of the product by simple filtration of the polymer matrix, easy handling, low moisture susceptibility, minimum side reaction, and recyclability of the polymer matrix for repeated use have drawn huge attention from industry and academia. ${ }^{29}$ Although triphenylphosphine is considered one of the worst atom-economic reagents due to its high carbon content, polymer-bound triphenylphosphine is getting a lot of applications in recent years mainly because of the speed and simplicity in the operation. ${ }^{30}$ The commonly encountered problems in solution-phase chemistry involving triphenylphosphine, such as removal of excess triphenylphosphine, triphenylphosphine complexes, and the by-product triphenylphosphine oxide can be overcome easily with polymer-bound triphenylphosphine. Moreover, for the reactions where polymer-bound triphenylphosphine acts as an oxygen-acceptor, the byproduct triphenylphosphine oxide can be reduced to triphenylphosphine by treatment with trichlorosilane. ${ }^{31}$

\section{Results and Discussion}

Continuing of our ongoing research interest in developing novel methodologies in organic synthesis, in particular, solid phase synthesis ${ }^{32}$ and keeping in mind the irony of the existing literature for the synthesis of alkyl halides, especially mono-halogenation, herein, we report a simple and efficient procedure for the iodination of alcohols using a combination of polymer-bound triphenylphosphine and iodine assisted protocol employing 'click' chemistry (Scheme 1). We have used organic polymers in order to simplify product isolation, so that reactions require only filtration, extraction and solvent removal for product purification.

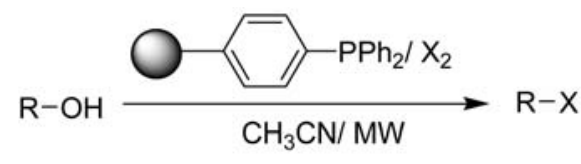

$\mathrm{R}=$ allylic, benzylic or other $1^{\circ}$ group

$\mathrm{X}=\mathrm{I}, \mathrm{Br}$

Scheme 1. Synthesis of alkyl halide

The iodination of benzyl alcohol was selected for optimization of the reaction conditions. Initially, the conversion of benzyl alcohol to benzyl iodide with polymer bound triphenylphosphine and iodine assisted by microwave in the presence of various solvents such as diethyl ether, ethyl acetate, $n$-hexane, acetone and dichloromethane at reflux temperature was studied. Among the various solvents tested for the reactions, acetonitrile was found to be the best solvent for these transformations. Henceforth, the iodination of various benzylic, allylic and aliphatic alcohols in the presence of polymer-bound triphenylphosphine (PB-TPP) and iodine in anhydrous acetonitrile was studied further. A variety of alcohols were smoothly converted to the corresponding iodides using this approach. The generality of the method was examined using aryl, allyl, alkyl alcohols and the results of the iodination reactions are summarized in Table 1.

It was observed that benzylic alcohols are highly reactive under our reaction conditions and yields were relatively less dependent on the substitution pattern on the phenyl rings (Table 1, entries 1-14, 22 and 26). However, we observed that primary alcohols converted to their corresponding iodides at a faster rate as compared to the secondary alcohols (Table 1, entries 23-25). Under the optimized conditions we also tried chemoselective conversion of butan-1,3-diol to its corresponding iodide. To our pleasure, the reaction gave exclusively 4-iodobutane-2-ol leaving one alcoholic group intact (Table 1, entry 21). Furthermore, 2-methylpent-2,4-diol gave 4-iodo-2-methylpentan-2-ol (Table 1, entry 24) which clearly showed the preference of secondary over tertiary substitution. Next, to test the scope and limitation of this methodology, we employed this reagent system in bromination by replacing the iodine with bromine. We were pleased to obtain the desired bromides in excellent yields within a very short time (Table 1, entries 9, 12). But, ironically, our attempt to convert phenol to its corresponding iodide bears no fruit (Table 1, entry 27).

Selectivity of the reaction system is always a subject of interest for synthetic organic chemist in contemporary chemistry. Since the invention of solid-phase chemistry by Merrifield in $1963,{ }^{33}$ initially for peptide synthesis, numerous literature reported that in solid phase synthesis site-selective reaction is very much feasible without any requirements for dilution of reaction mixture as the polymer beads are discrete (site-isolated) and reactions between 
Table 1. Microwave-assisted conversion of alcohols to halides ${ }^{\mathrm{a}, \mathrm{b}}$

$$
\begin{aligned}
& \mathrm{R}-\mathrm{OH} \frac{\mathrm{CH}-\mathrm{X}}{\mathrm{R}=\text { allylic, benzylic or other } 1^{\circ} \text { group }} \\
& \mathrm{X}=\mathrm{I}, \mathrm{Br}
\end{aligned}
$$

\begin{tabular}{|c|c|c|c|c|}
\hline Entry & $\mathbf{R}$ & Product & Time (min) & Yield $(\%)^{\mathrm{b}}$ \\
\hline 1. & & & 4 & 93 \\
\hline 2. & & & 4 & 93 \\
\hline 3. & & & 4 & 96 \\
\hline 4. & & & 3 & 91 \\
\hline 5. & & & 3 & 89 \\
\hline 6. & & & 4 & 79 \\
\hline 7. & & & 4 & 96 \\
\hline 8. & & & 4 & 92 \\
\hline 9. & & & 5 & 91 \\
\hline 10. & & & 4 & 95 \\
\hline 11. & & & 4 & 85 \\
\hline 12. & & & 5 & 92 \\
\hline 13. & & & 4 & 85 \\
\hline 14. & & $\mathrm{Br}$ & 4 & 87 \\
\hline 15. & & & 4 & 81 \\
\hline
\end{tabular}




\begin{tabular}{|c|c|c|c|c|}
\hline Entry & $\mathbf{R}$ & Product & Time (min) & Yield $(\%)^{b}$ \\
\hline 16. & & & 7 & 79 \\
\hline 17. & & & 4 & 92 \\
\hline 18. & & & 4 & 89 \\
\hline 19. & & & 4 & 79 \\
\hline 20. & & & 3 & 87 \\
\hline 21. & & & 3 & 88 \\
\hline 22. & & & 3 & 84 \\
\hline 23. & & & 5 & 91 \\
\hline 24. & & $\mathrm{OH}$ & 5 & 79 \\
\hline 25. & & & 5 & 76 \\
\hline 26. & & & 3 & 85 \\
\hline 27. & & & 5 & 0 \\
\hline
\end{tabular}

${ }^{\mathrm{a}}$ Molar ratio of alcohol/ PB-TPP/ $\mathrm{X}_{2}=1: 1.2: 1$

${ }^{\mathrm{b}}$ Unless otherwise mentioned reactions were carried out at $120{ }^{\circ} \mathrm{C}$ under MW

${ }^{\text {c }}$ Yields refer to pure isolated products, which were characterized by comparison of their physical and spectral data (IR, ${ }^{1} \mathrm{H}$ and ${ }^{13} \mathrm{C}$ NMR, MS) with authentic samples.

two polymer beads are impossible, thereby addressing the problem of polymerization. ${ }^{34}$ This concept stemmed from the hyperentropic character (high dilution principle) of the solid-phase method.${ }^{35}$ Recently, Firouzabadi et al. ${ }^{36}$ has reported a highly selective method for the iodination of alcohol using $\mathrm{ZrCl}_{4} / \mathrm{NaI}$ system obtaining as high as $97 \%$ mono-iodination product, whereas a similar reaction conduc- ted in the presence of $\mathrm{CeCl}_{3} \cdot 7 \mathrm{H}_{2} \mathrm{O} / \mathrm{NaI}$ produced the same product in $83 \%$ yield after $48 \mathrm{~h}$ as described by Deo et al. ${ }^{37}$

However, to the best of our knowledge, there is no report in literature of a selective mono-iodination of symmetrical diols in solid phase synthesis. Taking the high dilution theory into account, we assumed that if the in situ formation of activated oxo-phosphonium interme- 
Table 2. Mono-iodination of diols ${ }^{\mathrm{a}, \mathrm{b}}$

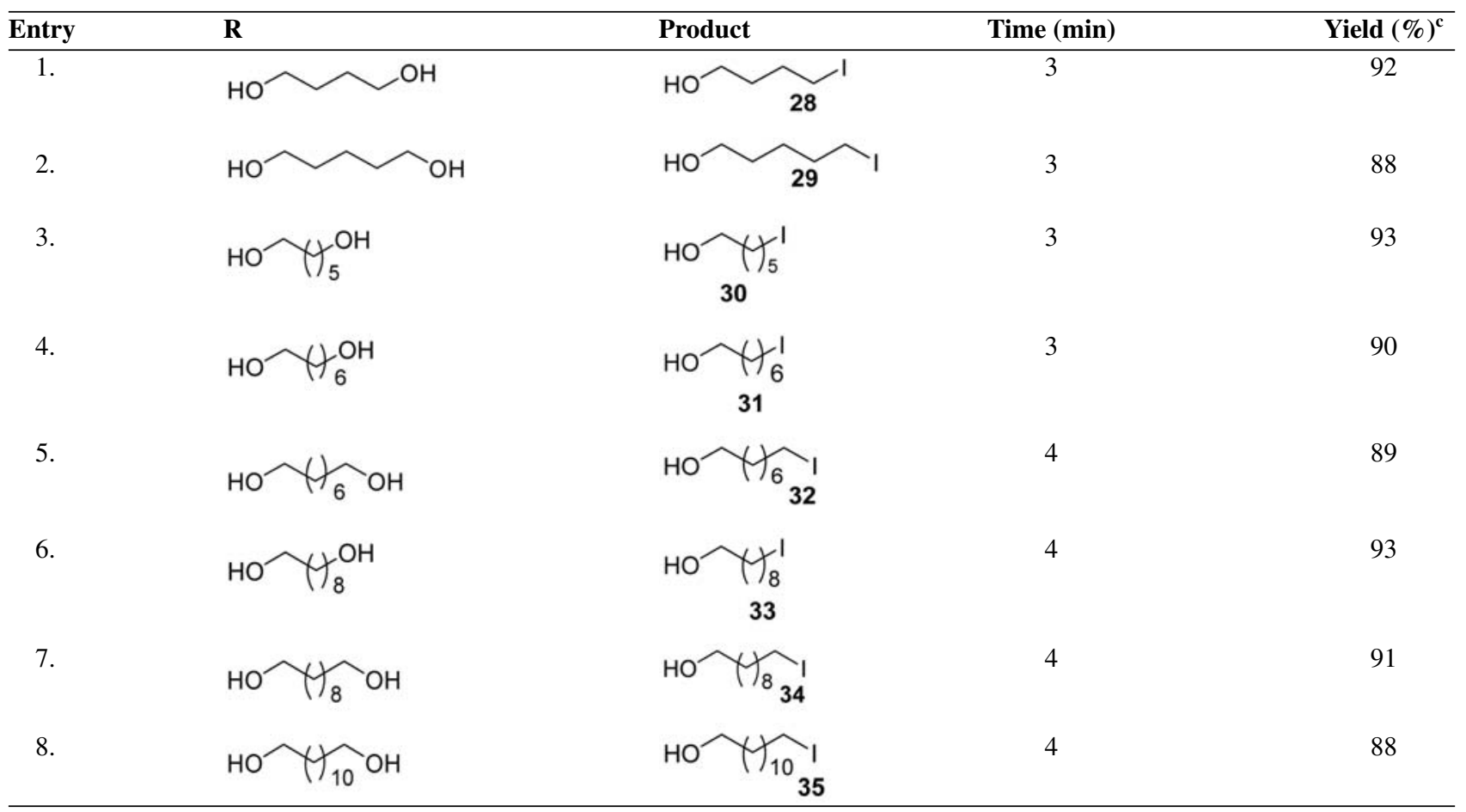

${ }^{\mathrm{a}}$ Molar ratio of alcohol/ $\mathrm{PB}-\mathrm{TPP} / \mathrm{I}_{2}=1: 1.2: 1$

${ }^{\mathrm{b}}$ Unless otherwise mentioned reactions were carried out at $120{ }^{\circ} \mathrm{C}$ under MW

${ }^{\mathrm{c}}$ Yields refer to pure isolated products and were characterized by comparison of their physical and spectral data (IR, ${ }^{1} \mathrm{H}$ and ${ }^{13} \mathrm{C}$ NMR, MS) with authentic samples.

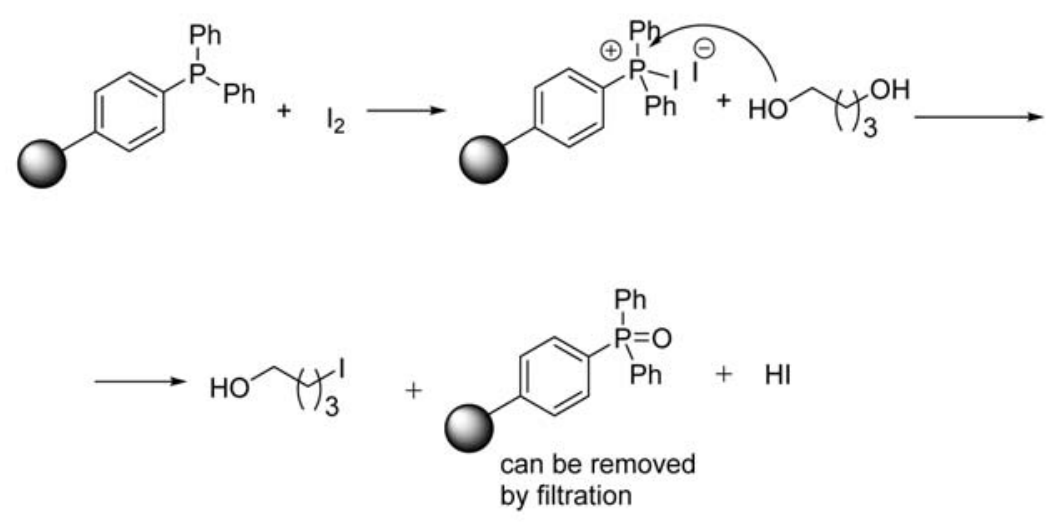

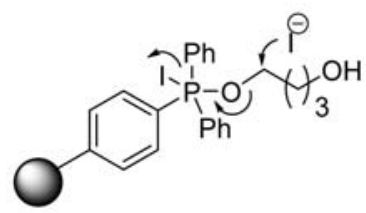

Scheme 2. Plausible mechanism for the mono-iodination of alcohols under optimized conditions

diate is followed by the nucleophilic attack by iodide anion would give us exclusively mono-iodide product without any requirement for dilution of the reaction system (Scheme 2). We first tested the iodination of 1,4-butanediol under our optimized conditions. To our contentment, the desired mono-iodide product was obtained in $92 \%$ yield (Table 3, entry 1 ). Inspired by this finding, we tried several mono-iodinations of symmetrical diols as given in Table 2.

\section{Conclusions}

In conclusion, a simple and highly chemoselective, site-selective process for the iodination of benzylic, allylic and aliphatic alcohols using polymer-bound triphenylphosphine in anhydrous acetonitrile assisted by microwave has been demonstrated. Use of polymer-supported reagents greatly simplifies product isolation requiring only simple filtration and solvent removal, whe- 
reas otherwise tedious column chromatography techniques are needed. Additionally, mono-halogenation products are obtained in high yield without any requirement of dilution of reaction mixture, which otherwise are very difficult to achieve. The mild reaction conditions, short reaction times, good to excellent yields and operational simplicity of the protocol are the advantages of this method.

\section{Experimental}

\section{1. General}

Milestones' Start SYNTH microwave was used for all the reactions. IR spectra were recorded on a Perkin-Elmer Spectrum One FTIR spectrometer. ${ }^{1} \mathrm{H}$ and ${ }^{13} \mathrm{C}$ NMR spectra were recorded on a Bruker $(500 \mathrm{MHz}$ and 400 $\mathrm{MHz}$ ) spectrometer using TMS as internal reference. Chemical shifts for ${ }^{1} \mathrm{H}$ NMR spectra are reported (in parts per million) relative to internal tetramethylsilane $\left(\mathrm{Me}_{4} \mathrm{Si}, \delta=\right.$ 0.0 ppm) with $\mathrm{CDCl}_{3}$ as solvent. ${ }^{13} \mathrm{C}$ NMR spectra were recorded at $125 \mathrm{MHz}$ and $100 \mathrm{MHz}$. Chemical shifts for ${ }^{13} \mathrm{C}$ NMR spectra are reported (in parts per million) relative to internal tetramethylsilane $\left(\mathrm{Me}_{4} \mathrm{Si}, \delta=0.0 \mathrm{ppm}\right)$ with $\mathrm{CDCl}_{3}$ as solvent. ${ }^{1} \mathrm{H}$ NMR data are reported in the order of chemical shift, multiplicity $(\mathrm{s}=$ singlet, $\mathrm{d}=$ doublet, $\mathrm{t}=$ triplet, $\mathrm{dd}=$ doublet of doublet, and $\mathrm{m}=$ multiplet), number of protons, and coupling constant in hertz (Hz). Mass spectra were obtained from Waters ZQ 4000 mass spectrometer by the ESI method, while the elemental analyses of the products were performed on a Perkin-Elmer-2400 $\mathrm{CHN} / \mathrm{S}$ analyzer. TLC plates were visualized by exposing in iodine chamber, UV-lamp or spraying with $\mathrm{KMnO}_{4}$ and heating.

\section{2. Typical Procedure for Iodination of Alcohol}

To a suspension of polymer-bound triphenylphosphine $(1.2 \mathrm{mmol})$ in anhydrous acetonitrile $(10 \mathrm{~mL})$ were added iodine $(1 \mathrm{mmol})$ and 1,6-hexanediol diol (1 $\mathrm{mmol})$. The reaction mixture was irradiated in microwave reactor at $120^{\circ} \mathrm{C}$ for $3 \mathrm{~min}$. The reaction mixture was filtered over a filter paper and washed with chloroform. The filtrate was extracted with aqueous sodium thiosulfate solution and dried with anhydrous sodium sulfate. Thereafter, solvent was removed under reduced pressure to obtain 6-iodohexan-1-ol (30) in $93 \%$ yield. ${ }^{1} \mathrm{H}$ NMR $\left(500 \mathrm{MHz}, \mathrm{CDCl}_{3}\right) \delta 3.65(\mathrm{t}, J=5 \mathrm{~Hz}, 2 \mathrm{H}), 3.20(\mathrm{t}, J=5$ $\mathrm{Hz}, 2 \mathrm{H}), 2.05(\mathrm{~s}, 1 \mathrm{H}), 1.84(\mathrm{q}, J=5 \mathrm{~Hz}, 2 \mathrm{H}), 1.58(\mathrm{q}, J=$ $10 \mathrm{~Hz}, 2 \mathrm{H}), 1.42(\mathrm{~m}, J=5 \mathrm{~Hz}, 4 \mathrm{H}) .{ }^{13} \mathrm{C}$ NMR $(125 \mathrm{~Hz}$, $\left.\mathrm{CDCl}_{3}\right) \delta 62.79,33.41,32.48,30.25,24.74,7.04 . \mathrm{IR}$ $(\mathrm{KBr}) \vee 3021,2400,1898,1598,1422,1381,1128,993$, 932, 813, 561, $455 \mathrm{~cm}^{-1}$. ESI-MS $\mathrm{m} / z 228.05\left(\mathrm{M}^{+}\right)$. Anal. Calcd for $\mathrm{C}_{6} \mathrm{H}_{13} \mathrm{IO}$ : C 31.60, H 5.75. Found: C 31.58, H 5.77 .

\section{2. 1. Spectroscopic Data of the Compounds}

Benzyl iodide (1) Yellow crystalline solid, $\mathrm{mp} 22-23{ }^{\circ} \mathrm{C}$ (lit. $\left.{ }^{17} \mathrm{mp} 21-23{ }^{\circ} \mathrm{C}\right) .{ }^{1} \mathrm{H}$ NMR $\delta 7.34(\mathrm{~d}, J=4.8 \mathrm{~Hz}, 2 \mathrm{H})$, $7.27(\mathrm{t}, J=4.8 \mathrm{~Hz}, 2 \mathrm{H}), 7.20(\mathrm{t}, J=2.68 \mathrm{~Hz}, 1 \mathrm{H}), 4.42(\mathrm{~s}$, $2 \mathrm{H}) ;{ }^{13} \mathrm{C}$ NMR $\delta 139.34,128.89,128.82,127.95,77.47$, 76.04, 5.94. IR $(\mathrm{KBr}) \vee 2917,1589,1147,1050,830,639$ $\mathrm{cm}^{-1}$. ESI-MS $m / z 217.95\left(\mathrm{M}^{+}\right)$. Anal. Calcd for $\mathrm{C}_{7} \mathrm{H}_{7} \mathrm{I}: \mathrm{C}$ 38.56, H 3.24. Found: C 38.55, H 3.25.

4-Methyl benzyl iodide (2) Pale yellow liquid, ${ }^{1} \mathrm{HNMR}$ $\left(\mathrm{CDCl}_{3}, 400 \mathrm{MHz}\right) \delta 7.26(\mathrm{~d}, J=8 \mathrm{~Hz}, 2 \mathrm{H}), 7.08(\mathrm{~d}, J=8$ $\mathrm{Hz}, 2 \mathrm{H}), 4.45$ (s, 2H), $2.30(\mathrm{~S}, 3 \mathrm{H}) .{ }^{13} \mathrm{C} \mathrm{NMR}\left(\mathrm{CDCl}_{3}\right.$, $100 \mathrm{MHz}) \delta 137.81,136.28,129.54,128.63,21.25,6.22$. IR $(\mathrm{KBr}) \vee 3020,2920,1590,1455,1148,1049,832,641$ $\mathrm{cm}^{-1}$. ESI-MS m/z $231.95\left(\mathrm{M}^{+}\right)$. Anal. Calcd for $\mathrm{C}_{8} \mathrm{H}_{9} \mathrm{I}$ : C 41.41, H 3.91. Found: C 41.43, H 3.89.

4-Methoxybenzyl iodide (3) Pale yellow solid, mp 25-26 ${ }^{\circ} \mathrm{C}$ (lit. ${ }^{17} \mathrm{mp} 25-27{ }^{\circ} \mathrm{C}$ ). ${ }^{1} \mathrm{H}$ NMR $\left(400 \mathrm{MHz}, \mathrm{CDCl}_{3}\right) \delta$ $3.72(\mathrm{~s}, 3 \mathrm{H}), 4.49(\mathrm{~s}, 2 \mathrm{H}), 7.35(\mathrm{~d}, J=4.8 \mathrm{~Hz}, 2 \mathrm{H}), 7.63$ ( $\mathrm{d}, J=4.8 \mathrm{~Hz}, 2 \mathrm{H}) .{ }^{13} \mathrm{C}$ NMR $\left(100 \mathrm{MHz}, \mathrm{CDCl}_{3}\right) \delta 6.19$, $55.19,113.35,128.49,131.62,158.13$. IR $(\mathrm{KBr}) \vee 3022$, $2915,1591,1478,1284,1221,1127,811,710 \mathrm{~cm}^{-1}$. ESIMS $m / z 247.97\left(\mathrm{M}^{+}\right)$. Anal. Calcd for $\mathrm{C}_{8} \mathrm{H}_{9} \mathrm{IO}: \mathrm{C} 38.74, \mathrm{H}$ 3.66. Found: C 38.76, H 3.64.

4-Chlorobenzyl iodide (8) Colorless crystalline solid, $\mathrm{mp}$ 58-59 ${ }^{\circ} \mathrm{C}$ (lit. $\left.{ }^{17} \mathrm{mp} 58-60{ }^{\circ} \mathrm{C}\right) .{ }^{1} \mathrm{H}$ NMR $(500 \mathrm{MHz}, \mathrm{CDC}-$ $\left.1_{3}\right) \delta 7.31(\mathrm{~d}, J=10 \mathrm{~Hz}, 2 \mathrm{H}), 7.26(\mathrm{~d}, J=5 \mathrm{~Hz}, 2 \mathrm{H}), 4.41$ (s, 2H). ${ }^{13} \mathrm{C}$ NMR $\left(125 \mathrm{~Hz}, \mathrm{CDCl}_{3}\right) \delta 137.87,133.62$, $129.48,128.29,4.19$. IR (KBr) v 3010, 2915, 1605, 1505, 1490, 1410, 1205, 1150, 1080, 825, $665 \mathrm{~cm}^{-1}$. ESI-MS $\mathrm{m} / \mathrm{z} 251.89\left(\mathrm{M}^{+}\right)$. Anal. Calcd for $\mathrm{C}_{7} \mathrm{H}_{6} \mathrm{ClI}$ : C 33.30, $\mathrm{H}$ 2.41. Found: C 33.28, H 2.39.

4-Bromobenzyl iodide (11) Crystalline white solid, $\mathrm{mp}$ 58-59 ${ }^{\circ} \mathrm{C}$ (lit. $\left.{ }^{21} \mathrm{mp} 57-59{ }^{\circ} \mathrm{C}\right) .{ }^{1} \mathrm{H}$ NMR $(500 \mathrm{MHz}$, CDC$\left.1_{3}\right) \delta 7.43(\mathrm{~d}, J=5 \mathrm{~Hz}, 2 \mathrm{H}), 7.25(\mathrm{~d}, J=10 \mathrm{~Hz}, 2 \mathrm{H}), 4.41$ $(\mathrm{s}, 2 \mathrm{H}) .{ }^{13} \mathrm{C}$ NMR $\left(125 \mathrm{~Hz}, \mathrm{CDCl}_{3}\right) \delta 138.39,132.01$, $130.42,121.78,4.46$. IR (KBr) v 3015, 2893, 1600, 1510, $1475,1398,1212,1131,1067,776,651,532,469 \mathrm{~cm}^{-1}$. ESI-MS $\mathrm{m} / z 295.81\left(\mathrm{M}^{+}\right)$. Anal. Calcd for $\mathrm{C}_{7} \mathrm{H}_{6}$ BrI: C 28.31, H 2.04. Found: C 28.31, H 2.06.

(2-Iodoethyl)benzene (15) Yellow liquid, ${ }^{1} \mathrm{H}$ NMR (400 $\left.\mathrm{MHz}, \mathrm{CDCl}_{3}\right) \delta 7.22(\mathrm{~m}, 3 \mathrm{H}), 7.16(\mathrm{t}, J=6.8 \mathrm{~Hz}, 2 \mathrm{H}), 3.30$ $(\mathrm{t}, J=8 \mathrm{~Hz}, 2 \mathrm{H}), 3.13(\mathrm{t}, J=7.6 \mathrm{~Hz}, 2 \mathrm{H}) .{ }^{13} \mathrm{C}$ NMR $(100$ $\left.\mathrm{MHz}, \mathrm{CDCl}_{3}\right) \delta 140.67,128.49,128.08,127.14,126.92$, 126.59, 40.40, 5.80; ESI MS m/z $231.96\left(\mathrm{M}^{+}\right)$. Anal. Calcd for $\mathrm{C}_{8} \mathrm{H}_{9} \mathrm{I}: \mathrm{C}$ 41.41, H 3.91. Found: C 41.42, H 3.90.

1-Iodooctadecane (16) White crystalline solid, mp $105-107{ }^{\circ} \mathrm{C} .{ }^{1} \mathrm{H}$ NMR $\left(400 \mathrm{MHz}, \mathrm{CDCl}_{3}\right) \delta 0.81(\mathrm{t}, J=$ $6.6 \mathrm{~Hz}, 3 \mathrm{H}), 1.19(\mathrm{~m}, 24 \mathrm{H}), 1.32(\mathrm{~m}, 2 \mathrm{H}), 1.75(\mathrm{~m}, 2 \mathrm{H})$, $3.10(\mathrm{t}, J=7.04 \mathrm{~Hz}, 2 \mathrm{H}) .{ }^{13} \mathrm{C} \mathrm{NMR}\left(100 \mathrm{MHz}, \mathrm{CDCl}_{3}\right) \delta$ $7.01,14.20,22.77,28.65,29.46,29.65,30.61,32.01$, 
33.66. IR $(\mathrm{KBr}) \vee 2912,2817,1438,1376,983,846,752$, $683 \mathrm{~cm}^{-1}$. ESI-MS $\mathrm{m} / z, 380.20\left(\mathrm{M}^{+}\right)$. Anal. Calcd for $\mathrm{C}_{18} \mathrm{H}_{37} \mathrm{I}$ : C 56.83, H 9.80. Found: C 56.84, H 9.79.

1-Iodooctane (17) Colorless liquid, ${ }^{1} \mathrm{H}$ NMR $(400 \mathrm{MHz}$, $\left.\mathrm{CDCl}_{3}\right) \delta 3.17(\mathrm{t}, J=6.8 \mathrm{~Hz}, 2 \mathrm{H}), 1.78(\mathrm{~m}, 2 \mathrm{H}), 1.36(\mathrm{t}, J$ $=6.4 \mathrm{~Hz}, 2 \mathrm{H}), 1.27(\mathrm{~s}, 4 \mathrm{H}), 0.87(\mathrm{t}, J=6.8 \mathrm{~Hz}, 3 \mathrm{H}) .{ }^{13} \mathrm{C}$ NMR $\left(100 \mathrm{MHz}, \mathrm{CDCl}_{3}\right) \delta 33.57,30.52,29.71,29.10$, 28.52, 22.64, 14.10, 7.39. IR (KBr) v 2421, 1822, 1573, 1454, 1214, 944, 913, 863, 712, $452 \mathrm{~cm}^{-1}$. ESI-MS $\mathrm{m} / \mathrm{z}$ $240.02\left(\mathrm{M}^{+}\right)$. Anal. Calcd for $\mathrm{C}_{8} \mathrm{H}_{17} \mathrm{I}$ : C 40.02, H 7.14. Found: C 40.01, H 7.15.

1-Iododecane (18) Colorless liquid, ${ }^{1} \mathrm{H}$ NMR $(500 \mathrm{MHz}$, $\left.\mathrm{CDCl}_{3}\right) \delta 3.18(\mathrm{t}, J=10 \mathrm{~Hz}, 2 \mathrm{H}), 1.81(\mathrm{q}, J=10 \mathrm{~Hz}, 2 \mathrm{H})$, $1.55(\mathrm{~s}, 2 \mathrm{H}), 1.38(\mathrm{~m}, J=5 \mathrm{~Hz}, 10 \mathrm{H}), 0.87(\mathrm{t}, J=5 \mathrm{~Hz}$, $3 \mathrm{H}) .{ }^{13} \mathrm{C}$ NMR $\left(125 \mathrm{~Hz}, \mathrm{CDCl}_{3}\right) \delta 33.59,31.91,30.52$, $29.70,29.62,29.55,29.43,29.34,28.55,22.69,14.13$, 7.36. IR (KBr) v $2422,1812,1577,1455,1217,947,915$, $865,710,458 \mathrm{~cm}^{-1}$. ESI-MS $\mathrm{m} / z 296.13\left(\mathrm{M}^{+}\right)$. Anal. Calcd for $\mathrm{C}_{12} \mathrm{H}_{25} \mathrm{I}$ : C $48.65, \mathrm{H}$ 8.51. Found: $\mathrm{C} 48.64, \mathrm{H}$ 8.49 .

Citronellyl iodide (19) Colorless liquid, ${ }^{1} \mathrm{H}$ NMR (400 $\left.\mathrm{MHz}, \mathrm{CDCl}_{3}\right) \delta 0.94(\mathrm{~d}, J=7.5 \mathrm{~Hz}, 3 \mathrm{H}), 1.21(\mathrm{~m}, 2 \mathrm{H})$, $1.56(\mathrm{~m}, 1 \mathrm{H}), 1.62(\mathrm{~s}, 3 \mathrm{H}), 1.70(\mathrm{~s}, 3 \mathrm{H}), 1.87(\mathrm{~m}, 2 \mathrm{H})$, $2.06(\mathrm{~m}, 2 \mathrm{H}), 3.18(\mathrm{t}, J=1.0 \mathrm{~Hz}, 2 \mathrm{H}), 5.10(\mathrm{t}, J=1.3 \mathrm{~Hz}$, $1 \mathrm{H}) .{ }^{13} \mathrm{C}$ NMR $\left(100 \mathrm{MHz}, \mathrm{CDCl}_{3}\right) \delta 5.33,18.70,18.79$, $24.56,25.34,33.90,36.51,40.92,124.49,131.38$. IR $(\mathrm{KBr}) \vee 2931,2411,1513,1423,1353,1278,994,766$, $701 \mathrm{~cm}^{-1}$. ESI-MS $\mathrm{m} / z, 266.06\left(\mathrm{M}^{+}\right)$. Anal. Calcd for $\mathrm{C}_{10} \mathrm{H}_{19} \mathrm{I}: \mathrm{C} 45.13, \mathrm{H}$ 7.20. Found: C 45.14, H 7.19.

Iodocyclohexane (25) Light yellow semi liquid, ${ }^{1} \mathrm{H}$ NMR $\left(400 \mathrm{MHz}, \mathrm{CDCl}_{3}\right) \delta 4.27(\mathrm{~m}, 1 \mathrm{H}), 2.15(\mathrm{~d}, J=9.6 \mathrm{~Hz}$, 2H) $2.00(\mathrm{~d}, J=14.4 \mathrm{~Hz}, 2 \mathrm{H}), 1.92(\mathrm{t}, J=12.8 \mathrm{~Hz}, 2 \mathrm{H})$, $1.60(\mathrm{t}, J=20 \mathrm{~Hz}, 2 \mathrm{H}), 1.39(\mathrm{~d}, J=8 \mathrm{~Hz}, 2 \mathrm{H}) .{ }^{13} \mathrm{C} \mathrm{NMR}$ $\left(100 \mathrm{MHz}, \mathrm{CDCl}_{3}\right) \delta 39.60,32.85,27.32,25.20$. IR (KBr) v 2419, 1820, 1575, 1455, 1224, 954, 916, 865, 715, 455 $\mathrm{cm}^{-1}$. ESI-MS $\mathrm{m} / z 210.00\left(\mathrm{M}^{+}\right)$. Anal. Calcd for $\mathrm{C}_{6} \mathrm{H}_{11} \mathrm{I}: \mathrm{C}$ 34.31, H 5.28. Found: C 34.33, H 5.26.

Diphenylmethyl iodide (26) Colorless crystalline solid; mp 68-70 ${ }^{\circ} \mathrm{C}$, (lit. $\left.{ }^{17} \mathrm{mp} 69-70{ }^{\circ} \mathrm{C}\right) .{ }^{1} \mathrm{H}$ NMR $(400 \mathrm{MHz}$, $\left.\mathrm{CDCl}_{3}\right) \delta 7.25(\mathrm{t}, J=7.2 \mathrm{~Hz}, 5 \mathrm{H}), 7.16(\mathrm{~d}, J=7.6 \mathrm{~Hz}, 5 \mathrm{H})$, $3.96(\mathrm{~s}, 1 \mathrm{H}) .{ }^{13} \mathrm{C} \mathrm{NMR}\left(100 \mathrm{MHz}, \mathrm{CDCl}_{3}\right) \delta 141.16$, $128.99,128.83,128.52,126.12,125.80,41.98 . \mathrm{IR}(\mathrm{KBr})$ v 3031, 2911, 1663, 1308, 1191, 1019, 745, $702 \mathrm{~cm}^{-1}$. ESI-MS m/z $293.97\left(\mathrm{M}^{+}\right)$. Anal. Calcd for $\mathrm{C}_{13} \mathrm{H}_{11} \mathrm{I}$ : C 53.09, H 3.77. Found: C 53.10, H 3.78.

5-Iodopentan-1-ol (29) Yellow liquid, ${ }^{1} \mathrm{H}$ NMR (400 $\left.\mathrm{MHz}, \mathrm{CDCl}_{3}\right) \delta 3.51(\mathrm{t}, J=7.6 \mathrm{~Hz}, 2 \mathrm{H}), 3.17(\mathrm{t}, J=6.8$ $\mathrm{Hz}, 2 \mathrm{H}), 2.05(\mathrm{~s}, 1 \mathrm{H}), 1.81(\mathrm{~m}, 2 \mathrm{H}), 1.48(\mathrm{~m}, 2 \mathrm{H}), 1.25$ $(\mathrm{m}, 2 \mathrm{H}) .{ }^{13} \mathrm{C}$ NMR $\left(100 \mathrm{MHz}, \mathrm{CDCl}_{3}\right) \delta 63.05,33.52$, $30.47,25.70,7.45$. IR (KBr) v 3612, 2431, 1834, 1563,
$1445,1210,949,918,868,718,457 \mathrm{~cm}^{-1}$. ESI-MS $\mathrm{m} / \mathrm{z}$ $213.99\left(\mathrm{M}^{+}\right)$. Anal. Calcd for $\mathrm{C}_{5} \mathrm{H}_{11} \mathrm{IO}$ : C 28.06, H 5.18. Found: C 28.04, H 5.20.

10-Iododecan-1-ol (34) Colorless liquid, ${ }^{1} \mathrm{H}$ NMR (400 $\left.\mathrm{MHz}, \mathrm{CDCl}_{3} \mathrm{TMS}\right) \delta 5.28(\mathrm{~s}, 1 \mathrm{H}), 3.62,(\mathrm{t}, J=6.8 \mathrm{~Hz}$, $2 \mathrm{H}), 3.17(\mathrm{t}, J=6.8 \mathrm{~Hz}, 2 \mathrm{H}), 1.78(\mathrm{~m}, 2 \mathrm{H}), 1.52(\mathrm{~m}, 2 \mathrm{H})$, $1.29(\mathrm{~d}, J=2.8 \mathrm{~Hz}, 12 \mathrm{H}) .{ }^{13} \mathrm{C} \mathrm{NMR}\left(100 \mathrm{MHz}, \mathrm{CDCl}_{3}\right) \delta$ $63.05,33.52,32.75,30.47,29.47,29.36,29.33,28.51$, 25.70, 7.45. IR (KBr) v 3611, 3011, 2405, 1888, 1598, $1425,1391,1129,998,945,815,564,458 \mathrm{~cm}^{-1}$. ESI-MS $m / z 284.07\left(\mathrm{M}^{+}\right)$. Anal. Calcd for $\mathrm{C}_{10} \mathrm{H}_{21} \mathrm{IO}: \mathrm{C} 42.27, \mathrm{H}$ 7.45. Found: C 42.26, H 7.45.

\section{Acknowledgements}

The authors gratefully acknowledge Science and Engineering Research Board (SERB)-DST, Govt. of India, New Delhi for financial supports (sanctioned no: SB/FT/CS-103/2013, SB/EMEQ-076/2014). The analytical services provided by Sophisticated Analytical Instrumentation Facility (SAIF), Indian Institute of Technology Madras, India is highly appreciated. D. D. thanks NIT Silchar for providing research fellowship.

\section{References}

1. (a) F. L. M. Pattison, J. B. Stothers, R. G. Woolford, J. Am. Chem. Soc. 1956, 78, 2255-2259.

http://dx.doi.org/10.1021/ja01591a062

(b) S.-K. Kang, W.-S. Kim, B.-H. Moon, Synthesis 1985, 12, 1161-1162. http://dx.doi.org/10.1055/s-1985-31464

2. N. Gupta, G. L. Kad, J. Singh, J. Mol. Catal. A: Chem. 2009, 302, 11-14. http://dx.doi.org/10.1016/j.molcata.2008.11.040

3. (a) R. Bohlmann. In Comprehensive Organic Synthesis; B. M. Trost, I. Fleming, Eds.; Pergamon Press: Oxford, 1991; Vol. 6, pp 203-223.

http://dx.doi.org/10.1016/B978-0-08-052349-1.00157-8

(b) R. D. Chambers, S. R. James. In Comprehensive Organic Chemistry; D. Barton, W. D. Ollis, Eds.; Pergamon Press: Oxford, 1979; Vol. 1, pp 493-575.

(c) M. -D. Su, S. Y. Chu, J. Am. Chem. Soc. 1999, 121, 10451058. http://dx.doi.org/10.1021/ja9831148

4. K. C. Nicolaou, N. Winssinger, D. Vourloumis, T. Ohshima, S. Kim, J. Pfefferkorn, J.-Y. Xu, T. Li, J. Am. Chem. Soc. 1998, 120, 10814-10826.

http://dx.doi.org/10.1021/ja9823870

5. W. Peng, B. S. J. Blaggs, Org. Lett. 2006, 8, 975-978. http://dx.doi.org/10.1021/ol060022b

6. J. Villieras, C. Bacquet, J. F. Normant, Bull. Chem. Soc. Fr. 1975, 1797.

7. Y. D. Vankar, C. T. Rao, Tetrahedron Lett. 1985, 26, 27172720. http://dx.doi.org/10.1016/S0040-4039(00)98145-0

8. M. Lauwers, B. Regnier, M. V. Eenoo, J. N. Denis, A. Krief, 
Tetrahedron Lett. 1979, 20, 1801-1804. http://dx.doi.org/10.1016/S0040-4039(01)86222-5

9. M. E. Jung, P. L. Ornstein, Tetrahedron Lett. 1977, 31, 26592662. http://dx.doi.org/10.1016/S0040-4039(01)83039-2

10. I. Fernandez, B. Garcia, S. Munoz, J. R. Pedro, R. Salud, Synlett 1993, 489-490.

http://dx.doi.org/10.1055/s-1993-22501

11. A. G. Martinez, R. M. Alvarez, E. T. Vilar, A. G. Fraile, J. O. Barnica, M. R. Hanack, L. Subramanian, Tetrahedron Lett. 1987, 28, 6441-6442. http://dx.doi.org/10.1016/S0040-4039(00)96882-5

12. H. Stone, H. Shechter, Org. Synth. 1963, 4, 323.

13. G. A. Olah, S. C. Narang, B. G. B. Gupta, R. Malhotra, J. Org. Chem. 1979, 44, 1247-1251. http://dx.doi.org/10.1021/jo01322a012

14. R. K. Haynes, M. Holden, Aust. J. Chem. 1982, 35, 517-524. http://dx.doi.org/10.1071/CH9820517

15. N. Iranpoor, H. Firouzabadi, G. Aghapour, A. R. Vaez zadeh, Tetrahedron 2002, 58, 8689-8693. http://dx.doi.org/10.1016/S0040-4020(02)01089-X

16. B. P. Bandgar, V. S. Sadavarte, L. S. Uppalla, Tetrahedron Lett. 2001, 42, 951-953. http://dx.doi.org/10.1016/S0040-4039(00)01953-5

17. S. Hayat, Atta-ur-Rahman, K. M. Khan, M. I. Choudhary, G. M. Maharvi, Zia-Ullah, E. Bayer, Synth. Commun. 2003, 33, 2531-2540. http://dx.doi.org/10.1081/SCC-120021844

18. A. R. Hajipour, A. Zarei, A. E. Ruoho, Synth. Commun. 2006, 36, 1039-1050. http://dx.doi.org/10.1080/00397910500503421

19. M. Tajbakhsh, R. Hosseinzadeh, Z. Lasemi, Synlett 2004, 635-638. http://dx.doi.org/10.1055/s-2004-815445

20. (a) G. Anilkumar, H. Nambu, Y. Kita, Org. Proc. Res. Dev. 2002, 6, 190-191. (b) L. Rokhum, C. Vanlalveni, R. Lalfakzuala, IJETCAS 2013, 4, 370-376. http://dx.doi.org/10.1021/op010094c

21. A. R. Hajipour, A. R. Falahati, A. E. Ruoho, Tetrahedron Lett. 2006, 47,4191-4196. http://dx.doi.org/10.1016/j.tetlet.2006.04.036

22. P. J. Garegg, R. Johansson, C. Ortega, G. J. Samuelsson, Chem. Soc., Perkin Trans 1. 1982, 681-683. http://dx.doi.org/10.1039/p19820000681

23. P. Tundo, P. Venturello, Synthesis 1979, 952-954. http://dx.doi.org/10.1055/s-1979-28885

24. P. Lidström, J. Tierney, B. Wathey, J. Westman, Tetrahedron 2001, 57, 9225-9283. http://dx.doi.org/10.1016/S0040-4020(01)00906-1

25. H. W. P. N'Dongo, I. Ott, R. Gust, U. Schatzschneider, J. Organomet. Chem. 2009, 694, 823-827. http://dx.doi.org/10.1016/j.jorganchem.2008.07.031

26. V. Cerezo, M. Amblard, J. Martinez, P. Verdie, M. Planas, L. Feliu, Tetrahedron 2008, 64, 10538-10545. http://dx.doi.org/10.1016/j.tet.2008.08.077

27. Y. Wang, R. L. Miller, D. R. Sauer, S. W. Djuric, Org. Lett. 2005, 7, 925-928. http://dx.doi.org/10.1021/ol050007r

28. (a) U. Boas, J. Brask, K. J. Jensen, Chem. Rev. 2009, 109, 2092-2118. http://dx.doi.org/10.1021/cr068206r (b) A. A. Thompson, J. A. Ellman, Chem. Rev. 1996, 96, 555-572. http://dx.doi.org/10.1021/cr9402081

(c) P. H. H. Hermkens, H. C. J. Ottenheim, D. Rees, Tetrahedron 1996, 53, 5643-5678.

(d) F. Balkenhohl, C. von dem Bussche-Hünnefeld, A. Lansky, C. Zechel, Angew. Chem., Int. Ed. Engl. 1996, 35, 2288-2337. http://dx.doi.org/10.1002/anie.199622881 http://dx.doi.org/10.1016/S0040-4020(97)00279-2

29. (a) J. J. Araya, G. Montenegro, L. A. Mitscher, B. N. Timmermann, J. Nat. Prod. 2010, 73, 1568-1572.

http://dx.doi.org/10.1021/np100465h

(b) A. M. Harned, S. Mukherjee, D. L. Flynn, P. R. Hanson, Org. Lett. 2003, 5, 15-18.

http://dx.doi.org/10.1021/o10270327

(c) S. Mothana, N. Chahal, S. Vanneste, D. G. Hall, J. Comb. Chem. 2007, 9, 193-196.

http://dx.doi.org/10.1021/cc060149s

(d) J. P. Nandy, M. Prakesch, S. Khadem, R. P. Thirupathi, U. Sharma, P. Arya, Chem. Rev. 2009, 109, 1999-2060.

http://dx.doi.org/10.1021/cr800188v

30. (a) S. S. Michaelidou, P. A. Koutentis, Tetrahedron 2009, 65, 8428-8433. http://dx.doi.org/10.1016/j.tet.2009.07.064

(b) C. Delvare, C. S. Harris, L. Hennequin, P. Koza, C. Lambert-van der Brempt, J. Pelleter, O. Willerval, ACS Comb. Sci. 2011, 13, 449-452.

http://dx.doi.org/10.1021/co200062n

(c) A. El-Dahshan, Ahsanullah, J. Rademann, Pept. Sci. 2010, 94, 220-228. http://dx.doi.org/10.1002/bip.21378

(d) I. C. Christoforou, A. S. Kalogirou, P. A. Koutentis, Tetrahedron 2009, 65, 9967-9972.

http://dx.doi.org/10.1016/j.tet.2009.10.003

(e) A. C. Comely, S. E. Gibson, N. J. Hales, Chem. Commun. 2000, 305-306. http://dx.doi.org/10.1039/a909462h

(f) A. R. Tunoori, D. Dutta, G. I. Georg, Tetrahedron Lett. 1998, 39, 8751-8754.

http://dx.doi.org/10.1016/S0040-4039(98)01988-1

(g) B. Classon, Z. Liu, J. Org. Chem. 1988, 53, 6126-6130. http://dx.doi.org/10.1021/jo00261a032

(h) T. Holletz, D. Cech, Synthesis 1994, 789-791. http://dx.doi.org/10.1055/s-1994-25575

31. S. L. Regen, D. P. Lee, J. Org. Chem. 1975, 40, 1669-1670. http://dx.doi.org/10.1021/jo00899a042

32. (a) L. Rokhum, G. Bez, J. Chem. Sci. 2011, 124, 687-691. http://dx.doi.org/10.1007/s12039-012-0241-5

(b) L. Rokhum, G. Bez, Canadian J. Chem. 2013, 91, 300-306. http://dx.doi.org/10.1139/cjc-2012-0164

(c) L. Rokhum, G. Bez, Tetrahedron Lett. 2013, 54, 5500-5509. http://dx.doi.org/10.1016/j.tetlet.2013.07.146

(d) G. Pathak, L. Rokhum, ACS Comb. Sci. 2015, 17, 483-487. http://dx.doi.org/10.1021/acscombsci.5b00086

33. R. B. Merrifield, J. Am. Chem. Soc. 1963, 85, 2149-2154. http://dx.doi.org/10.1021/ja00897a025

34. (a) B. M. Trost, R. W. Warner, J. Am. Chem. Soc. 1982, 104, 6112-6114. http://dx.doi.org/10.1021/ja00386a045

(b) K. C. Nicolaou, J. Pastor, N. Winssinger, F. Murphy, J. Am. Chem. Soc. 1998, 120, 5132-5133. 
http://dx.doi.org/10.1021/ja980675q

35. (a) J. I. Crowley, H. Rapoport, J. Am. Chem. Soc. 1970, 92, 6363-6365. http://dx.doi.org/10.1021/ja00724a059

(b) J. I Crowley, H. Rapoport, Acc. Chem. Res. 1976, 9, 135-144. http://dx.doi.org/10.1021/ar50100a003
36. H. Firouzabadi, N. Iranpoor, M. Jafarpour, Tetrahedron Lett. 2004, 45, 7451-7454. http://dx.doi.org/10.1016/j.tetlet.2004.08.056

37. M. Di Deo, E. Marcantoni, E. Torregiani, G. Bartoli, M. C. Bellucci, M. Bosco, L. Sambri, J. Org. Chem. 2000, 65, 2830-2833. http://dx.doi.org/10.1021/jo991894c

\section{Povzetek}

Predstavljamo uspešno in visokoselektivno metodo za pretvorbo alilnih, benzilnih in alifatskih alkoholov v ustrezne halide s pomočjo trifenilfosfina, vezanega na polimerni nosilec, ob prisotnosti joda pod pogoji obsevanja $\mathrm{z}$ mikrovalovi. V primeru simetričnih diolov, smo z visokimi izkoristki pripravili mono-jodirane produkte. Poleg tega smo pri našem postopku opazili visoko regioselektivnost. Odlike našega pristopa so enostavnost izvedbe, brez potrebe po kolonski kromatografiji za čiščenje produktov, možnost recikliranja uporabljenih reagentov, kratki reakcijski časi in odlični izkoristki. Večina funkcionalnih skupin pri teh pretvorbah ostane nespremenjenih. 